\title{
Editorial
}

Jinghai Li*

\section{Towards a new paradigm of chemical engineering}

https://doi.org/10.1515/revce-2018-0037

In the past 40 years, chemical engineering research in China has made significant progress, not only in developing industrial technology but also in fundamental research. Particularly in the last decade, several important chemical technologies have been commercialized, such as coal to olefins (CTO), methanol to olefins (MTO), maximizing iso-paraffins process (MIP) and so on. The proportion of chemical engineering publications has increased from $7.5 \%$ of the world total to $15.2 \%$, ranking it the second in the world. Such a leap has been driven by economic development and government investment in science and technology. Now facing new challenges in sustainable development and the shift of the science and technology landscape and paradigm, the China chemical engineering community has been discussing the strategy for the future, with a totally different methodology from that of 40 years ago. These background features include: an increase of the industrial research and development (R\&D) capability, emerging demands from sustainable development goals (SDGs), interdisciplinary with other disciplines, and possible landscape and paradigm shifts due to significantly improved data, supercomputing, virtual reality, and generally, information technology.

These considerations were reflected in the National Guideline on Medium- and Long-Term Program for Science and Technology Development (http://www.most.gov.cn/ mostinfo/xinxifenlei/gjkjgh/200811/t20081129_65774. htm), and implemented, respectively, by the (Ministry of Science and Technology (MOST), the National Natural Science Foundation of China (NSFC), the Ministry of Finance (MOF), the Ministry of Education (MOE), the Chinese Academy of Sciences (CAS) and other sectors. Some of these goals have been accomplished, and others are still in progress. This two-part special issue of Reviews in Chemical Engineering (part 2 is scheduled to be published in January 2020) includes some chemical engineering representative works in China, reflecting partially the current landscape of chemical engineering research, but, of course, with the legacy of previous generations.

*Corresponding author: Jinghai Li, Institute of Process Engineering, Chinese Academy of Sciences, Beijing 100190, P.R. China,

e-mail: jhli@ipe.ac.cn
Historically, contemporary chemical engineering research in China was initiated by a group of pioneers educated in Western countries in the early 1920s with the educational methodology of unit operations and then in the 1950s with that of transport phenomena. The core knowledge base for chemical engineering was outlined as mass-, momentum-, energy-transfer and reaction, that is, the so-called “三传一反” in Chinese. On the other hand, due to the separation among different industries administrated by the governmental ministries of industries, this chemical-engineering know how was distributed among various research institutions serving different industries. This mode of chemical engineering education and research has advantages in supporting the initial development of various industries, but limits its extension to the common knowledge base of others. Other fields, such as food, pharmaceutical, energy and metallurgy, also established independent research and education systems, based on chemical engineering principles although the communication and cooperation between them were very limited.

An example of which I am very familiar, is metallurgical engineering research. For instance, the Institute of Chemical Metallurgy of the CAS was created by a famous metallurgist Professor Chu-Phay Yep, together with a chemical engineer, Professor Mooson Kwauk and a hydro metallurgist, Professor Jiayong Chen. This enabled the institute to intensify metallurgical processes by using chemical engineering principles. This made the institute unique, but not recognized sufficiently by the chemical engineering community as its research and activities before the 1980s were mainly within the field of metallurgy. After China opened up in the 1980s, and particularly, after the dissolution of various governmental industrial ministries in the 1990s, the constraints from ministries disappeared, motivating natural interaction and cooperation among different engineering disciplines. For example, the Institute of Chemical Metallurgy was renamed as the Institute of Process Engineering in 2001 in order to extend its scope from chemical engineering in metallurgy to the whole spectrum of process engineering.

Another limitation in the development of chemical engineering has been the separation between the chemistry research and that of chemical engineering. These two disciplines have been working separately though the 
situation has changed recently, such as in the development of MTO technology.

The chemical engineering community in China is currently facing both global and domestic challenges in the transition from the resource-based and investment-driven economy into the "innovation-driven" and sustainable economy. Meanwhile, the emerging new paradigm of science and technology, driven by information technology, big data, supercomputing, and virtual reality etc. is generating many opportunities.

Responding to these challenges and opportunities, the Chinese chemical engineering community is taking further actions. Among many different efforts, the main funding agency of fundamental research, the National Natural Science Foundation of China (NSFC), has given priority to chemical engineering frontier research by launching a major program on mesoscience entitled: "Mechanism and manipulation at mesoscales in multiphase reaction processes" (http://www.mesoscience. org/), to promote transdisciplinary interaction between mathematics, chemistry, physics, material, chemical engineering, metallurgy, energy, and others. This program, launched in 2012, focuses on various mesoscale problems at two different levels of chemical engineering, that is, interfacial and material structures at the material level, and dynamic heterogeneous structures at the reactor level. The program is covered by almost all mainstream universities and institutions in the field. On the other hand, the co-funding mechanisms were established to support the study of industrial interest to promote the transformation of development mode, such as joint program with industries and local governments. These actions have motivated and supported research on fundamental issues, which are oriented directly to demands for future development.

This two-part special issue includes a collection of chemical engineering research topics in China and reviews of the developments and the current progress in this field. The authors are members of top departments and institutes of the chemical engineering community in China, and many are the winners of the National Science Fund for Distinguished Young Scholars. The articles cover the current landscape of chemical engineering, consisting of four categories: - Industrial technologies commercialized in recent years through a long-term fundamental research, such as coal chemistry;

- Principles and methodologies in chemical engineering in extending to different fields, such as the multiscale approach, process system engineering, multiphase mass transfer, and material;

- Upgraded traditional processes in resource utilization, such as mineral processing, heavy oil processing and membrane technology;

- Emerging topics such as ionic liquids and biotechnology.

The way to a new paradigm is facing the tendency of open and global science, transdisciplinarity, and international cooperation in coping with global challenges. Integrating knowledge and global resources is critical to this change. I hope that this special issue will catalyze this cooperation.

I thank the editors Prof. Dan Luss and Prof. Neima Brauner who accepted my proposal to edit this special issue, and helped in the editing process. In addition, they coordinated the agreement between the Chemical Industry Press in China and De Gruyter to publish the issue in both English and Chinese so that Chinese industrial people can read the volume easily. Thanks are also extended to all authors and reviewers for their efforts to produce high quality papers and reviews. I apologize to the invited authors whose papers could not be included due to the deadline limitation, which will be hopefully published in the Chinese version. 